\title{
Trajectories of self-rated health in the last 15 years of life by cause of death
}

\section{Citation}

Stenholm, Sari, Mika Kivimäki, Marja Jylhä, Ichiro Kawachi, Hugo Westerlund, Jaana Pentti, Marcel Goldberg, Marie Zins, and Jussi Vahtera. 2015. "Trajectories of Self-Rated Health in the Last 15 Years of Life by Cause of Death." European Journal of Epidemiology 31 (2): 177-85. https://doi.org/10.1007/s10654-015-0071-0.

\section{Permanent link}

http://nrs.harvard.edu/urn-3:HUL.InstRepos:41275577

\section{Terms of Use}

This article was downloaded from Harvard University's DASH repository, WARNING: This file should NOT have been available for downloading from Harvard University's DASH repository.

\section{Share Your Story}

The Harvard community has made this article openly available.

Please share how this access benefits you. Submit a story.

Accessibility 


\title{
Trajectories of self-rated health in the last $\mathbf{1 5}$ years of life by cause of death
}

\author{
Sari Stenholm ${ }^{1,2} \cdot$ Mika Kivimäki ${ }^{3,4,5} \cdot$ Marja Jylhä $^{2}$ \\ Ichiro Kawachi $^{6} \cdot$ Hugo Westerlund $^{7} \cdot$ Jaana Pentti $^{4}$. \\ Marcel Goldberg ${ }^{8} \cdot$ Marie Zins $^{8} \cdot$ Jussi Vahtera $^{1,4,9}$
}

Received: 25 February 2015/ Accepted: 10 July 2015/Published online: 23 July 2015

(C) Springer Science+Business Media Dordrecht 2015

\begin{abstract}
Poor self-rated health is associated with increased risk of mortality, but no previous study has examined how long-term trajectories of self-rated health differ among people at risk of subsequent death compared to those who survive. Data were drawn from French occupational cohort (the GAZEL study, 1989-2010). This nested case-control study included 915 deceased men and women and 2578 controls matched for sex, baseline age, occupational grade and marital status. Self-rated health was measured annually and dichotomized into good versus poor health. Trajectories of poor self-rated health up to 15 years were compared among people who subsequently died to those who survived. Participants contributed to an average
\end{abstract}

Electronic supplementary material The online version of this article (doi:10.1007/s10654-015-0071-0) contains supplementary material, which is available to authorized users.

Sari Stenholm

sari.stenholm@utu.fi

1 Department of Public Health, University of Turku, 20014 Turku, Finland

2 School of Health Sciences and the Gerontology Research Center (GEREC), University of Tampere, Tampere, Finland

3 Department of Epidemiology and Public Health, University College London, London, UK

4 Finnish Institute of Occupational Health, Helsinki, Finland

5 Department of Public Health, Faculty of Medicine, University of Helsinki, Helsinki, Finland

6 Department of Society, Human Development, and Health, Harvard School of Public Health, Boston, MA, USA

7 University of Stockholm, Stockholm, Sweden

8 INSERM, UMS 011, Hôpital Paul Brousse, Villejuif, France

9 Turku University Hospital, Turku, Finland
10.3 repeated assessments of self-rated health. Repeatedmeasures log-binomial regression analysis with generalized estimating equations showed an increased prevalence of poor self-rated health in cases 13-15 years prior to death from ischemic and other cardiovascular disease [multivariable-adjusted risk ratio $2.06,95 \%$ confidence interval (CI) 1.55-2.75], non-smoking-related cancers $(1.57,95 \%$ CI 1.30-1.89), and suicide (1.78, $95 \%$ CI 1.00-3.16). Prior to death from ischemic and other cardiovascular disease, increased rates of poor self-rated health were evident even among persons who were free of cardiovascular diseases (2.05, $95 \%$ CI 1.50-2.78). In conclusion, perceptions of health diverged between the surviving controls and the deceased already 15 years prior to death. For cardiovascular mortality, decline in self-rated health started before diagnosis of the disease leading to death. The findings suggest that declining self-rated health might capture pathological changes before and beyond the disease diagnosis.

Keywords Self-rated health - Cause-specific mortality . Trajectory $\cdot$ Cohort study

\section{Introduction}

First reports linking poor self-rated health with increased risk of mortality were published over three decades ago [1]. The strength of the association between poor self-rated health and mortality may vary depending on the specific cause of death [2-8]. The self-rated health-mortality association has been suggested to be stronger when the cause of death is due to a chronic condition, such as diabetes or coronary heart disease, in comparison to sudden causes of death such as accidents [5]. A limitation in this 
evidence, however, is that most studies have only assessed self-rated health at one point in time, ignoring the fact that self-rated health might fluctuate over the course of long follow-up periods. In our recent study based on repeated self-rated health measurements over time, we found that men and women who subsequently died rate their health as poor up to a decade prior to death compared to their surviving controls [9]. In that study cause-specific-mortality data were not available and thus it is not known whether self-rated health trajectories vary according to specific cause of death. A further limitation is that previous studies have not been able to determine the time order between the decline in self-rated health and diagnosis of the chronic disease leading to death. Thus, it is not known whether poor self-rated health follows the diagnosis leading to death or whether persons who were undiagnosed also have poor self-rated health.

In this retrospective study, we utilized cause-specific mortality data and annual measurements of self-rated health in a large French occupational cohort to examine trajectories of self-rated health up to 15 years prior to death. We sought to pinpoint when perceptions of health begin to diverge among people at risk of subsequent death compared to those who survive. In addition, we examined whether poor self-rated health predicted mortality even among those who were still undiagnosed of the disease related to the cause of death.

\section{Methods}

\section{Study population}

The GAZEL cohort was established in 1989 and comprises employees of the French national gas and electricity company: Electricité de France-Gaz de France (EDFGDF) [10]. At baseline, 20,624 employees (73\% men) aged 35-50 consented to participate. Information about health, lifestyle, and individual, familial, social, and occupational characteristics of the GAZEL participants is collected by annual surveys. Informed consent was obtained from all participants and the study received ethical approval from the French national ethics committee [Commission nationale de l'informatique et des libertés (CNIL)].

In this study we analyzed data from GAZEL participants who died between 2000 and 2010. We restricted inclusion in the study to those who had at least one self-reported health assessment $10-15$ years prior to death. Thus, the sample analyzed consisted of 774 men and 141 women who died at a mean age of 61.5 (SD 4.6). The analyses were based on a case-control design with the deceased persons being the cases and randomly selected 1-3 controls for each case. The average number of controls for each case was 2.8. Controls were matched for sex, baseline age, occupational grade and marital status, leading to 2188 male and 390 female controls. Self-reported health for each case and control was traced backwards for 15 years and followup for controls ended when their case died. Thus, cases and controls received roughly the same number of follow-up measurements. On average, cases provided data from 10.3 (SD 4.6) and controls from 11.4 (SD 4.1) of the possible 15 study waves.

\section{Assessment of self-rated health}

Self-rated health (SRH) was assessed by using a standard item included in the annual questionnaires sent to all participants between the years 1989-2010: "How would you judge the state of your general health?" The participants responded on an 8-point Likert scale $(1=$ very good, $8=$ very poor), which was dichotomized by categorizing response scores $1-4$ as good health and scores $5-8$ as poor health, as previously validated [11]. We used all available annual measurements in the 15-year timeframe before death.

\section{Mortality outcome}

Mortality data on all participants were obtained annually from EDF-GDF as it pays out retirement benefits. In the analysis we included deaths that occurred between 1 January 2000 and 31st December 2010. Cause of death was obtained from the French National Death Index. Causespecific mortality was coded using the International Classification of Diseases, Tenth Revision (ICD-10). We distinguished between deaths due to ischemic heart disease and cerebrovascular disease (I20-I25 or I46 or I60-I69), other cardiovascular disease (I00-I15 or I26-I45 or I47I59 or I70-I99), smoking-related cancers [C00-C06 (lip and oral cavity) or C09-C15 (pharynx) or C25 (biliary tract) or C30-C34 (respiratory organs) or C38 (heart) or C64-C68 (urinary tract)], other cancer (C00-D48 excluding above-mentioned smoking-related cancers), suicides (E95 or X60-X84), other external causes (E80-E94 or E96-E99 or S00-T98 or V01-X59 or X85-Y98) and other deaths.

\section{Covariate assessment}

The matching procedure made deceased and surviving controls comparable at baseline. However, since occupational grade and marital status can change during the follow-up and since we also wanted to compare individuals dying from different causes, the analyses were further adjusted for residual confounding factors-including age at 
death, sex, latest occupational grade, marital status and baseline lifestyle factors.

Occupational grade was derived from EDF-GDF records and was classified into three grades: (1) high (managers), (2) intermediate (technical staff, line managers, and administrative associate professionals), and (3) low (clerical and manual workers), on the basis of categorizations from the French National Institute of Statistics and Economic Studies. Marital status was dichotomized into (1) not married or cohabiting and (2) married or cohabiting. The latest values for occupational grade and marital status were utilized and they were considered as potential confounders or mediators in the association between SRH and mortality. The values for controls were obtained from the corresponding study wave.

Of the lifestyle factors were included smoking status, alcohol use [12], body mass index (BMI) derived from selfreported body weight and height and physical activity [13]. The baseline or first available measurement for both deceased and surviving controls were used and these lifestyle factors were considered as potential confounders in the association between SRH and mortality.

To take into account chronic diseases potentially affecting on SRH evaluation, we utilized information from the annual questionnaire which included a checklist of various conditions. Participants were asked to endorse all the health problems they are suffering currently or in the past. The following diseases related to causes of death were utilized: hypertension, angina pectoris, myocardial infarction, stroke, cancer and depression. This approach has been validated against medically certified sickness absence records, indicating some under-reporting of diagnoses, which, nonetheless, is less pronounced for severe and disabling diseases such as those studied here-with the exception of hypertension [14]. Information on diseases was treated as time-varying and they were obtained from the same time point as the SRH. We considered that participants had a particular chronic disease if they reported that disease in the year in question or had reported that disease in any of the prior years.

\section{Statistical analyses}

Characteristics of the study population are presented by cause of death. The values for controls were selected from the same study wave as for the cases.

Trajectories of poor SRH in the 15 years were assessed using log-binominal regression analysis with generalized estimating equations controlling for the intra-individual correlation between repeated measurements using an exchangeable correlation structure $[15,16]$. Interaction effect of sex and SRH on mortality was examined, but found to be non-significant $(p=0.26)$, thus results are presented for men and women combined and adjusting for sex. Due to the higher prevalence of poor SRH in women, we however, present the sex-specific results in the supplemental material (Supplementary eFigure 1).

We plotted SRH trajectories for all deceased cases and surviving controls as well as for deceased cases by cause of death using quadratic equations for time prior to death. To examine when the prevalence of poor SRH among the deceased cases (overall and cause-specific) and surviving controls began to diverge during the 15 -year period, we contrasted SRH at 3-year intervals between the cases and controls $(15-13,12-10,9-7,6-4$ and 3-1 years prior to death). In addition, to examine the overall change in SRH over 15 year of follow-up, we compared the first SRH measurement $(-15)$ with the last measurement $(-1)$ within cases and controls. The analysis was initially adjusted for age and sex. Thereafter models were further adjusted for the latest occupational grade and marital status. The models were also adjusted for baseline BMI, smoking, alcohol and physical activity.

Finally, to separate the effect of chronic diseases and SRH trajectories prior death, we repeated the main analysis by including only person observations as long as these persons were free of diseases related to their cause of death. For cardiovascular diseases we considered angina pectoris, myocardial infarction and stroke and for cancer we considered cancer. Results are presented as risk ratios (RR) and their $95 \%$ confidence intervals (CI). The SAS 9.4 Statistical Package was used for all analyses (SAS Institute Inc., Cary, NC).

\section{Results}

The average age at death was 61.5 (SD 4.6) years among deceased cases and the respective age of the controls was 61.2 (SD 4.5) years. Characteristics of deceased cases and surviving controls are shown in Table 1.

The mean SRH score (1-8) ranged from 3.46 (SD 1.48) at 15 years prior to death to 4.43 (SD 1.77) at 1 year prior to death among deceased and from 3.14 (SD 1.30) to 3.23 (SD 1.20) among survivors. The distribution of SRH across the 15-year period among descendants and survivors is shown in Supplementary eFigure 1. The prevalence of poor SRH (scores 5-8) across the 15-year period was higher among deceased cases compared to controls ranging from 1.5-fold (RR 1.57, $95 \%$ CI 1.37-1.80) 13-15 years prior to death to 2.5 -fold ( $R R$ 2.47, $95 \%$ CI 2.20-2.76) 1-3 years prior to death (Fig. 1; Table 2). Adjustment for occupational grade and baseline lifestyle factors had only a small effect on the association between SRH and all-cause deaths. Sex-specific results for all-cause mortality are shown in Supplement Material (Supplementary eFigure 2 


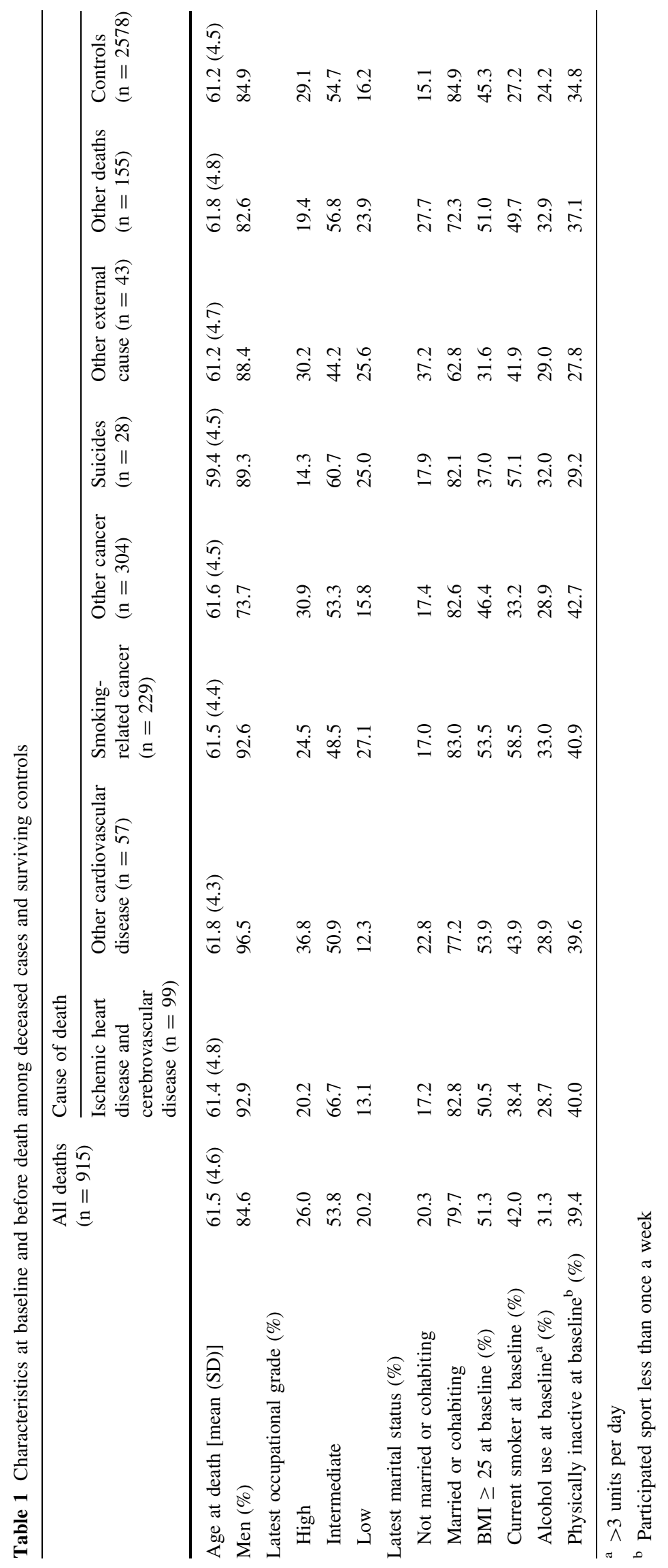




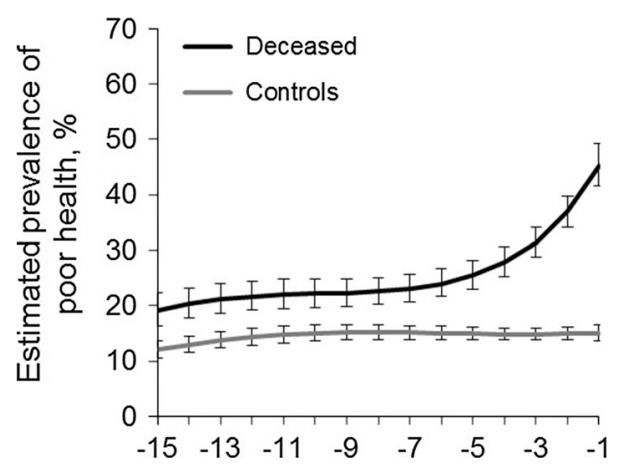

Fig. 1 Estimated prevalence with $95 \%$ confidence intervals of poor self-rated health during the 15 years prior to death in deceased cases and surviving controls

and eTable 1) indicating higher prevalence of poor SRH among women in the years preceding the death.

Figure 2 shows the trajectories of poor SRH in the 15 years preceding death by cause of death. Compared to surviving controls, those dying from ischemic heart disease or cerebrovascular disease reported poorer SRH throughout the 15 years prior to death and prevalence was more than twofold already $13-15$ years prior to death (RR 2.15, $95 \%$ CI 1.61-2.88) (Table 2). Among those dying from other cardiovascular diseases, the prevalence of poor SRH was began to increase $12-10$ years prior to death (RR 1.67, $95 \%$ CI 1.20-2.34) and increased nearly threefold (RR 2.82, $95 \%$ CI 2.08-3.84) 1-3 years before death compared to surviving controls. Occupational grade, marital status and baseline life-style factors did not influence on the association between SRH and deaths due to cardiovascular diseases. We repeated the analysis by including only observations as long as these persons were free of angina pectoris, myocardial infarction and stroke. We found that in this subgroup those dying from ischemic heart disease or cerebrovascular disease still reported poorer SRH 9-15 years prior to death compared to surviving controls (Table 3). In addition, those dying from other cardiovascular diseases reported increasingly poor SRH with approaching death compared to surviving controls. When comparing deaths due to cardiovascular disease, those dying from other cardiovascular diseases showed nearly two-fold higher prevalence of poor SRH in the proximity to death (RR 1.78, $95 \%$ 1.11-2.87) than those dying from ischemic heart disease or cerebrovascular disease (Supplementary eTable 2).

Among those dying from smoking-related cancer, the trajectory of poor SRH among cases generally tracked that of surviving controls, increasing drastically only in the 1-3 year window prior to death (Fig. 2; Table 2). Compared to surviving controls, among those dying from nonsmoking-related cancers the likelihood of poor SRH was
Table 2 Risk ratios (RR) and their $95 \%$ confidence interval (CI) for poor self-rated health comparing deceased cases to surviving controls at different time points prior to death by causes of death

\begin{tabular}{|c|c|c|c|c|c|c|}
\hline \multirow{2}{*}{$\begin{array}{l}\text { Cause of death } \\
\text { Years prior to death }\end{array}$} & \multicolumn{3}{|c|}{ Model 1} & \multicolumn{3}{|c|}{ Model 2} \\
\hline & RR & \multicolumn{2}{|c|}{$95 \%$ CI } & RR & \multicolumn{2}{|c|}{$95 \% \mathrm{CI}$} \\
\hline \multicolumn{7}{|l|}{ All deaths } \\
\hline $15-13$ & 1.57 & 1.37 & 1.80 & 1.45 & 1.26 & 1.65 \\
\hline $12-10$ & 1.41 & 1.25 & 1.59 & 1.30 & 1.15 & 1.47 \\
\hline $9-7$ & 1.62 & 1.43 & 1.83 & 1.50 & 1.33 & 1.69 \\
\hline $6-4$ & 1.70 & 1.50 & 1.93 & 1.58 & 1.39 & 1.79 \\
\hline $3-1$ & 2.47 & 2.20 & 2.76 & 2.30 & 2.05 & 2.57 \\
\hline
\end{tabular}

Ischemic heart disease and cerebrovascular disease

$\begin{array}{lllllll}15-13 & 2.15 & 1.61 & 2.88 & 2.06 & 1.55 & 2.75 \\ 12-10 & 1.72 & 1.33 & 2.22 & 1.61 & 1.25 & 2.07 \\ 9-7 & 1.91 & 1.47 & 2.48 & 1.80 & 1.39 & 2.33 \\ 6-4 & 1.46 & 1.01 & 2.09 & 1.37 & 0.95 & 1.97 \\ 3-1 & 1.52 & 1.04 & 2.23 & 1.49 & 1.02 & 2.19\end{array}$

Other cardiovascular disease

$\begin{array}{lllllll}15-13 & 1.60 & 0.98 & 2.59 & 1.49 & 0.94 & 2.37 \\ 12-10 & 1.67 & 1.20 & 2.34 & 1.57 & 1.15 & 2.13 \\ 9-7 & 2.08 & 1.47 & 2.95 & 1.95 & 1.39 & 2.74 \\ 6-4 & 2.09 & 1.42 & 3.07 & 1.95 & 1.33 & 2.86 \\ 3-1 & 2.82 & 2.08 & 3.84 & 2.66 & 1.96 & 3.61\end{array}$

Smoking-related cancer

$\begin{array}{lllllll}15-13 & 1.21 & 0.94 & 1.57 & 1.04 & 0.80 & 1.34 \\ 12-10 & 1.09 & 0.85 & 1.40 & 0.94 & 0.74 & 1.20 \\ 9-7 & 1.29 & 1.02 & 1.63 & 1.12 & 0.88 & 1.42 \\ 6-4 & 1.29 & 1.02 & 1.63 & 1.12 & 0.88 & 1.42 \\ 3-1 & 2.21 & 1.84 & 2.65 & 1.91 & 1.59 & 2.30\end{array}$

Other cancer

\begin{tabular}{lllllll}
$15-13$ & 1.62 & 1.34 & 1.97 & 1.57 & 1.30 & 1.89 \\
$12-10$ & 1.36 & 1.13 & 1.64 & 1.30 & 1.09 & 1.56 \\
$9-7$ & 1.82 & 1.52 & 2.17 & 1.76 & 1.48 & 2.10 \\
$6-4$ & 1.82 & 1.52 & 2.17 & 1.76 & 1.48 & 2.10 \\
$3-1$ & 2.75 & 2.38 & 3.19 & 2.71 & 2.34 & 3.14 \\
Suicide & & & & & & \\
$15-13$ & 1.89 & 1.04 & 3.42 & 1.78 & 1.00 & 3.16 \\
$12-10$ & 1.18 & 0.60 & 2.30 & 1.14 & 0.62 & 2.07 \\
$9-7$ & 1.66 & 0.83 & 3.34 & 1.66 & 0.85 & 3.25 \\
$6-4$ & 1.66 & 0.83 & 3.34 & 1.66 & 0.85 & 3.25 \\
$3-1$ & 2.19 & 1.29 & 3.70 & 2.11 & 1.26 & 3.52 \\
Other external cause & & & & & & \\
$15-13$ & 1.72 & 1.08 & 2.73 & 1.57 & 0.99 & 2.50 \\
$12-10$ & 1.62 & 1.03 & 2.53 & 1.52 & 0.98 & 2.36 \\
$9-7$ & 1.69 & 1.02 & 2.81 & 1.57 & 0.95 & 2.59 \\
$6-4$ & 1.69 & 1.02 & 2.81 & 1.57 & 0.95 & 2.59 \\
$3-1$ & 1.94 & 1.20 & 3.14 & 1.84 & 1.13 & 2.99 \\
Other deaths & & & & & & \\
$15-13$ & 1.49 & 1.10 & 2.01 & 1.31 & 0.97 & 1.75 \\
$12-10$ & 1.65 & 1.29 & 2.11 & 1.46 & 1.15 & 1.86 \\
\hline
\end{tabular}


Table 2 continued

\begin{tabular}{|c|c|c|c|c|c|c|}
\hline Cause of death & Mod & & & Mod & & \\
\hline Years prior to death & $\mathrm{RR}$ & $95 \%$ & & $\mathrm{RR}$ & $95 \%$ & \\
\hline $9-7$ & 2.10 & 1.66 & 2.66 & 1.85 & 1.47 & 2.31 \\
\hline $6-4$ & 2.10 & 1.66 & 2.66 & 1.85 & 1.47 & 2.31 \\
\hline $3-1$ & 2.87 & 2.33 & 3.54 & 2.50 & 2.04 & 3.07 \\
\hline
\end{tabular}

Model 1 is adjusted for age and sex. Model 2 is additionally adjusted for latest occupational grade and marital status, and for the baseline BMI, smoking, alcohol use and physical inactivity
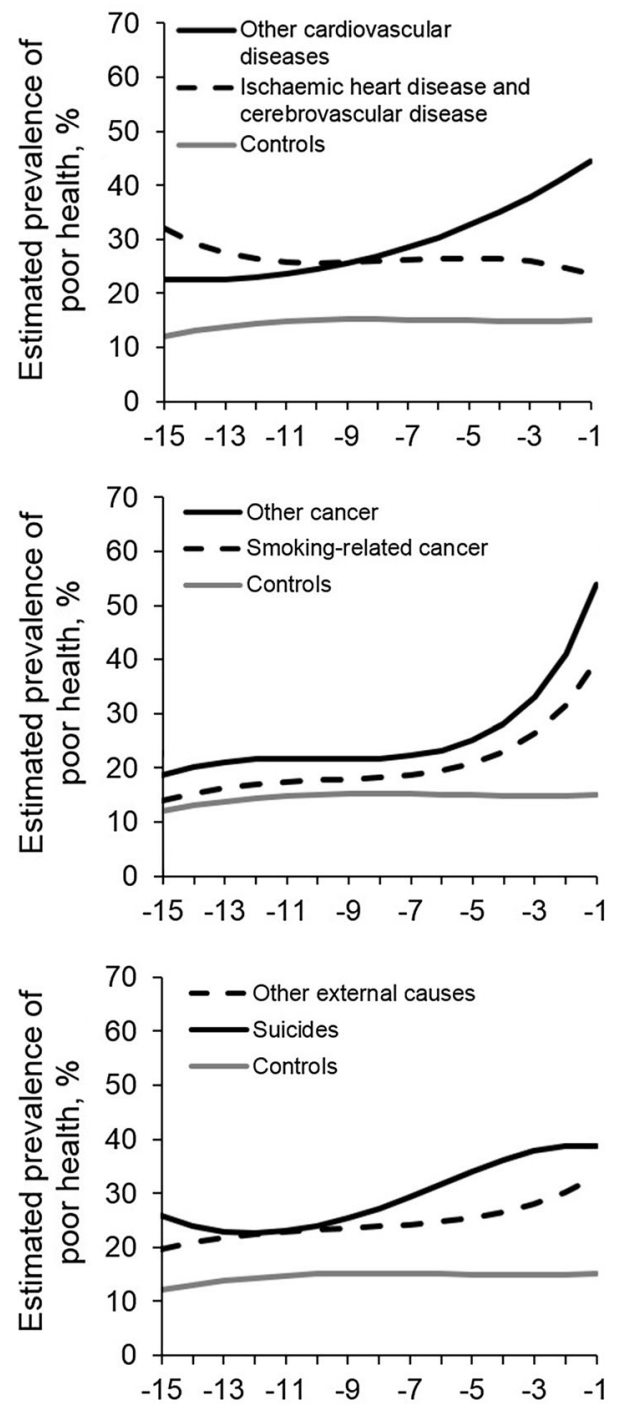

Fig. 2 Estimated prevalence of poor self-rated health during the 15 years prior to death in surviving controls and deceased cases according to different causes of death

1.6-fold already 13-15 years prior to death (RR 1.62, $95 \%$ CI 1.34-1.97) and increased to 2.7-fold 1-3 years prior to death (RR 2.75, $95 \%$ CI 2.38-3.19). Adjustment for occupational grade and marital status attenuated the association between SRH and deaths. Further adjustment for lifestyle factors had little effect on the strength of the association. After including only person observations as long as these persons were free of cancer, we found that those dying from smoking-related cancer and other cancers still reported poorer SRH in the 1-3 and 4-6 years prior to death compared to surviving controls, respectively (Table 3 ). When comparing deaths due to other cancer, those dying from smoking-related cancer showed 1.4-fold higher prevalence of poor SRH in the proximity to death (RR 1.42, $95 \% 1.15-1.74)$ than those dying from ischemic heart disease or cerebrovascular disease (Supplementary eTable 2).

Compared to surviving controls, those committing suicide reported poorer SRH throughout 15 years prior to death; the risk ratio was 1.9 (95\% CI 1.04-3.42) 15-13 years prior to death and almost the same 3-1 years prior to death (RR 2.19, $95 \%$ CI 1.29-3.70) (Fig. 2; Table 2). Adjustment for depression attenuated markedly these risk ratios and 3-1 years prior to death the SRH no longer significantly differed between deceased and surviving participants. Among those dying to other external causes, the risk ratios were 1.7 -fold $(95 \% 1.08-2.73)$ and (RR 1.94, $95 \%$ CI 1.20-3.14), respectively. Occupational grade and marital status explained part of the association between SRH and deaths due to non-suicidal external causes but baseline life-style factors had no effect on it. When comparing deaths due to external causes, there was no difference in poor SRH prevalence in any time point between those committing a suicide and those dying to other external causes (Supplementary eTable 2).

\section{Discussion}

In this large cohort of French employees followed up with annual surveys, we found that perceptions of health diverged between the surviving controls and the deceased already 15 years prior to death and the self-rated health trajectories varied according to causes of death. Those who died from any cause had 1.5 times higher prevalence of poor self-rated health already $13-15$ years prior to their death compared to age, sex and ethnicity matched surviving controls. Participants who subsequently died from ischemic cardiovascular or cerebrovascular disease were twice as likely to report poor self-rated health as the surviving controls. Importantly, this difference was evident even among those not diagnosed for cardio- or cerebrovascular disease. Increased risk of poor self-rated health were also found 13-15 years before fatal non-smokingrelated cancer and deaths from external causes and suicide. For non-ischemic cardiovascular disease this increase was observed 10-12 years prior to death the corresponding time 
Table 3 Risk ratio (RR) and their $95 \%$ confidence interval (CI) for poor self-rated health comparing deceased cases to surviving controls at different time points prior to death among subjects without specific diseases related to causes of death

\begin{tabular}{|c|c|c|c|c|c|}
\hline & $\begin{array}{l}\text { No of disease free } \\
\text { observations }\end{array}$ & $\begin{array}{l}\text { No of total } \\
\text { observations }\end{array}$ & $\mathrm{RR}$ & $95 \% \mathrm{CI}$ & \\
\hline \multicolumn{6}{|c|}{ Ischemic heart disease and cerebrovascular disease ${ }^{\mathrm{a}}$} \\
\hline $15-13$ & 155 & 173 & 2.05 & 1.50 & 2.78 \\
\hline $12-10$ & 208 & 241 & 1.34 & 1.00 & 1.81 \\
\hline $9-7$ & 180 & 215 & 1.50 & 1.10 & 2.06 \\
\hline $6-4$ & 144 & 185 & 1.18 & 0.74 & 1.86 \\
\hline $3-1$ & 126 & 182 & 0.98 & 0.54 & 1.77 \\
\hline \multicolumn{6}{|c|}{ Other cardiovascular disease ${ }^{\mathrm{a}}$} \\
\hline $15-13$ & 91 & 97 & 1.53 & 0.94 & 2.47 \\
\hline $12-10$ & 113 & 122 & 1.49 & 1.06 & 2.10 \\
\hline $9-7$ & 102 & 116 & 1.82 & 1.24 & 2.66 \\
\hline $6-4$ & 94 & 113 & 2.10 & 1.41 & 3.13 \\
\hline $3-1$ & 90 & 113 & 2.53 & 1.74 & 3.69 \\
\hline \multicolumn{6}{|c|}{ Smoking-related cancer ${ }^{\mathrm{b}}$} \\
\hline $15-13$ & 412 & 440 & 1.03 & 0.79 & 1.33 \\
\hline $12-10$ & 482 & 505 & 0.95 & 0.74 & 1.22 \\
\hline $9-7$ & 477 & 499 & 1.09 & 0.84 & 1.40 \\
\hline $6-4$ & 437 & 482 & 1.09 & 0.84 & 1.40 \\
\hline $3-1$ & 358 & 476 & 1.44 & 1.14 & 1.82 \\
\hline \multicolumn{6}{|c|}{ Other cancer ${ }^{\mathrm{b}}$} \\
\hline $15-13$ & 594 & 638 & 1.16 & 0.95 & 1.42 \\
\hline $12-10$ & 664 & 723 & 1.17 & 0.92 & 1.49 \\
\hline $9-7$ & 622 & 697 & 1.17 & 0.92 & 1.49 \\
\hline $6-4$ & 510 & 677 & 1.36 & 1.01 & 1.84 \\
\hline $3-1$ & 317 & 642 & 1.79 & 1.01 & 3.18 \\
\hline
\end{tabular}

Models are adjusted for age, sex, latest occupational grade and marital status, and for the baseline BMI, smoking, alcohol use and physical inactivity

${ }^{a}$ Excluding persons with angina pectoris, myocardial infarction and stroke

b Excluding persons with cancer point being 6 years prior to deaths from smoking-related cancer.

To our knowledge, this is the first study to describe selfrated health trajectories over 15 years prior to death by causes of death. Our results from all-cause deaths are consistent with a recent study based on the Health and Retirement Study showing that men and women who died at age of $\geq 65$ years and older perceived their health worse already 11-12 years prior to death, compared to their surviving controls [9].

Several previous studies have examined the association between poor self-rated health and cause-specific mortality, but these studies have relied on a single self-rated health measurement $[2-8,17]$ as opposite to 15 repeated measurements in the current study. The strongest association related to poor self-rated health has been observed with cancer mortality based on both short [3,5] and long followup studies [4]. A cohort study of Dutch elderly men found that the association remained statistically significant even after adjusting for baseline cancer [3], but the other studies did not consider baseline disease status in the analysis. Another often studied outcome is cardiovascular mortality and many shorter follow-up studies have observed an association between poor self-rated health and increased risk of cardiovascular mortality $[3,5,6]$, although in these studies the association did not remain significant after adjusting or excluding participants with index disease at baseline [3,6]. On the other hand, one large cohort study from Finland with 23 years of follow-up shows an association between poor self-rated health with cardiovascular mortality which remained statistically significant after adjustment for subject's medical history and cardiovascular disease risk factors [7].

In the current study, we used repeated measurement information on self-rated health and diagnosed diseases and were able to examine more closely the time order between the decline in self-rated health and being diagnosed for the chronic disease that led to death. In analysis of observations when the person was still not diagnosed with angina pectoris, myocardial infarction or stroke, we 
found that self-rated health was lower among deceased cases than surviving controls already $10-15$ years prior to cardiovascular deaths. After excluding person observations related to cancer diagnosis, self-rated health started to diverge between cases and controls 4-6 years prior to cancer death. This suggests that single-item self-rated health assessment might capture aspects of undiagnosed disease, including symptoms, feelings and bodily sensations. Information on the turning point in self-rated health trajectory before death might be important because it could help to recognize persons with declining health in a timely manner. However, given the presumably low specificity, it remains unclear whether this would allow identification of patients who would benefit from early clinical interventions.

A major strength of our study compared to previous studies is that we were able to compare self-rated health trajectories across different causes of death. One interesting finding was that those dying from ischemic heart disease and cerebrovascular disease had relatively constant but elevated levels of poor self-rated health throughout the 15 years prior to death, whereas among those dying from non-ischemic cardiovascular disease, poor self-rated health increased steadily with approaching death. Non-ischemic cardiovascular diseases may influence everyday life in many ways and person feels the disease with advancing condition and this is likely to be mirrored in worsening perceived health. Death from ischemic cardiovascular diseases-coronary heart disease and cerebrovascular diseases-can often be sudden and without a slowly advancing perception of worsening health status. Moreover, risk factors for ischemic cardiovascular diseases, such as high blood pressure, high blood sugar, obesity and atherosclerosis, often cluster and may be linked to many symptomatic chronic conditions and therefore a person may evaluate his/her health constantly as being somewhat suboptimal [18].

This study has many other strengths including repeated annual measurements of self-rated health over 15-year time period prior to death. This enabled us to identify the time point when self-rated health trajectories begin to diverge before death from those in surviving controls. The participation rate was high and on average the participants provided data on more than 10 of the possible 15 preceding years and only 3-5\% of participants had only one SRH measurement. In the examination of the loss of follow-up, those who responded only once were more likely to report poor SRH at baseline compared to those who had at least ten measurements. This suggests that the prevalence of poor SRH with approaching death may be underestimated in this study. Finally, by using a nested case-control design the confounding role of sex, age, occupational grade and marital status was taken care of.
This study also has some limitations. GAZEL is an occupational cohort and all participants have stable jobs and therefore the findings cannot extrapolate to the general population including unemployed individuals [19]. In addition, the study population was relatively young the average age of death being 61.5 years. Thus, the causes of death are likely to be different from those in populations aged 80 years and older. Furthermore, we were not able to examine all causes of deaths due to small number of events. For example, chronic pulmonary disease is important cause of death, especially among smokers, but there were only four cases dying from that cause. For the subgroup analysis we only included individuals who were free of chronic conditions. However, because these diseases were based on self-reports, we may have included some individuals who actually had the disease but were unaware of it or chose not to report it. We were also unable to control the precise date of disease diagnosis which hinders us to determine the precise temporal distance between disease and self-rated health in relation to mortality. Finally, there is potential problem of time-varying confounding, because new disease onset can be confounder and mediator of the relation between self-rated health and mortality. We tried to take this into account by conducting a subanalysis among those not diagnosed for cardio- or cerebrovascular disease or cancer, but the actual association between poor self-rated health and mortality may still be underestimated.

In conclusion, longitudinal modeling of annually repeated data showed that poor self-rated health predicts mortality up to 15 years prior to subsequent death and that self-rated health trajectories vary according to cause of death. The findings of this study suggest that declining self-rated health might capture pathological changes before and beyond the disease diagnosis. Further research is needed to determine whether self-rated health could be used to detect persons in high risk in a timely manner during the life course as regular monitoring of self-rated health during appointments would be easy to implement in health care practice.

Acknowledgments This work was supported by the EU's Era-Age 2 program (Academy of Finland 264944), Academy of Finland (286294) and the Swedish Research Council for Health, Working Life and Welfare (Forte, 2012-1661). Mika Kivimäki is supported by the Medical Research Council (K013351) and a professorial fellowship from the Economic and Social Research Council, UK. The funders had no role in study design, data collection and analysis, decision to publish, or preparation of the manuscript.

\section{Compliance with ethical standards}

Conflict of interest The authors disclose no conflict of interest.

Ethical standard This study was approved by the French national ethics committee [Commission nationale de l'informatique et des libertés (CNIL)] and informed consent was obtained from all participants. 


\section{References}

1. Mossey JM, Shapiro E. Self-rated health: a predictor of mortality among the elderly. Am J Public Health. 1982;72(8):800-8.

2. Tsuji I, Minami Y, Keyl PM, Hisamichi S, Asano H, Sato M, et al. The predictive power of self-rated health, activities of daily living, and ambulatory activity for cause-specific mortality among the elderly: a three-year follow-up in urban Japan. J Am Geriatr Soc. 1994;42(2):153-6.

3. Pijls LT, Feskens EJ, Kromhout D. Self-rated health, mortality, and chronic diseases in elderly men. The Zutphen study, 1985-1990. Am J Epidemiol. 1993;138(10):840-8.

4. Giltay EJ, Vollaard AM, Kromhout D. Self-rated health and physician-rated health as independent predictors of mortality in elderly men. Age Ageing. 2012;41(2):165-71.

5. Benjamins MR, Hummer RA, Eberstein IW, Nam CB. Self-reported health and adult mortality risk: an analysis of causespecific mortality. Soc Sci Med. 2004;59(6):1297-306.

6. Kaplan GA, Goldberg DE, Everson SA, Cohen RD, Salonen R, Tuomilehto $\mathrm{J}$, et al. Perceived health status and morbidity and mortality: evidence from the Kuopio ischaemic heart disease risk factor study. Int J Epidemiol. 1996;25(2):259-65.

7. Heistaro S, Jousilahti P, Lahelma E, Vartiainen E, Puska P. Self rated health and mortality: a long term prospective study in eastern Finland. J Epidemiol Community Health. 2001;55(4): 227-32.

8. Fernandez-Ruiz M, Guerra-Vales JM, Trincado R, Fernandez R, Medrano MJ, Villarejo A, et al. The ability of self-rated health to predict mortality among community-dwelling elderly individuals differs according to the specific cause of death: data from the NEDICES cohort. Gerontology. 2013;59(4):368-77.

9. Stenholm S, Pentti J, Kawachi I, Westerlund H, Kivimaki M, Vahtera J. Self-rated health in the last 12 years of life compared to matched surviving controls: the health and retirement study. PLoS One. 2014;9(9):e107879.

10. Goldberg M, Leclerc A, Bonenfant S, Chastang JF, Schmaus A, Kaniewski N, et al. Cohort profile: the GAZEL cohort study. Int J Epidemiol. 2007;36(1):32-9.

11. Niedhammer I, Chea M. Psychosocial factors at work and self reported health: comparative results of cross sectional and prospective analyses of the French GAZEL cohort. Occup Environ Med. 2003;60(7):509-15.

12. Zins M, Carle F, Bugel I, Leclerc A, Di Orio F, Goldberg M. Predictors of change in alcohol consumption among Frenchmen of the GAZEL study cohort. Addiction. 1999;94(3):385-95.

13. Sjösten N, Kivimäki M, Singh-Manoux A, Ferrie JE, Goldberg M, Zins $M$, et al. Change in physical activity and weight in relation to retirement: the French GAZEL cohort study. BMJ Open. 2012;2:e000522.

14. Metzger MH, Goldberg M, Chastang JF, Leclerc A, Zins M. Factors associated with self-reporting of chronic health problems in the French GAZEL cohort. J Clin Epidemiol. 2002;55(1): $48-59$.

15. Zeger SL, Liang K-Y. Longitudinal data analysis for discrete and continuous outcomes. Biometrics. 1986;42:121-30.

16. Diggle PJ, Liang KY, Zeger SL. Analysis of longitudinal data. Oxford: Oxford University Press; 1994.

17. DeSalvo KB, Bloser N, Reynolds K, He J, Muntner P. Mortality prediction with a single general self-rated health question. A meta-analysis. J Gen Intern Med. 2006;21(3):267-75.

18. Jylhä M. What is self-rated health and why does it predict mortality? Towards a unified conceptual model. Soc Sci Med. 2009;69(3):307-16.

19. Poncet M, Chevalier A, Bumsel F, Lahon G. Mortality among active workers at EDG-GDF: social and occupational disparities and evolution. Rev Epidemiol Sante Publique. 2003;51(5):481-91. 